\title{
Designs for Experiments with Large Number of Treatments
}

\author{
Thattil RO* \\ Professor Emeritus, University of Peradeniya, Sri Lanka
}

Submission: May 12, 2017; Published:May 25, 2017

*Corresponding author: Thattil RO, Professor Emeritus, University of Peradeniya, Sri Lanka, Email: thattil@pgia.ac.lk

\section{Introduction}

It is disheartening to note that many experimenters try to fit in their treatments in complete blocks even when the number of treatments is large. This may be mainly due to the lack of knowledge of incomplete block designs in general. There is no doubt that when homogenous blocks that can accommodate all the treatments are available, the Randomized Complete Block Design (RCBD) is one of the best designs that can be used. However, there are instances, where block size is limited by the very nature of the block itself. If the litter (natural block) of an animal is considered, the block size is limited to the size of the litter. Thus, R.C.B.D. is ruled out in such cases. In experimentation with animals such as rats and pigs where litter size of around 12 can be found, it cannot accommodate all treatments inside a Complete Block when the numbers of treatments exceed this number. In experiments with twins the block size is obviously two.

In field experiments such as varietal trials, it is usual to find the number of varieties (treatments) investigated to be sometimes as large as 40-50. In large factorial experiments using Complete Blocks is foolhardy. This may be the reason why some experimenters shy away from performing experiments with more than 3 factors [1].

\section{Incomplete Block Designs (IBD)}

When the number of treatments is large the alternative to the R.C.B.D. is the family of Incomplete Block Designs. In an I.B.D it is obvious that all treatments are not found in every block. In a general I.B.D. block size, number of replicates per treatment need not be the same. However, it is useful to have block sizes that are same and also to have equal number of replicates for each treatment. If the numbers of replicates are different, the precision of comparisons will be different for different pairs of treatments.

Among the Incomplete Block Designs the most useful design is the balanced Incomplete Block (BIB) design. In the BIB design the number of replicates per treatment is a constant, while the number of times a pair of treatments occur together in the blocks is also a constant, thus ensuring equal precision for all pairwise comparisons. The efficiency of the B.I.B. design compared to the R.C.B.D. is higher due to the reduction of block size.

When enough blocks cannot be found, it may not be possible to construct a B.I.B. design. In such cases, partial balance can yet be obtained, but the number of times a pair of treatments occurs together in the blocks will be different for different pairs. It is not useful to have too much of inbalance. However, Partially Balanced Incomplete Block (PBIB) designs with not more than 2 levels of precision can usually be constructed, in practice. It is left to the experimenter to decide which pairwise comparisons are more important. This information can be used at the design stage. Catalogues for I.B.D. are available in advanced texts on design and analysis of experiments. Analysis of data from I.B.D. is not a problem due to the availability of statistical software packages such as Statistical Analysis System (SAS) and Statistical package for Social Sciences (SPSS) [2].

\section{Lattice Designs}

As an alternative to IBD another class of designs namely Lattice Designs are especially useful to deal with the problem of testing a very large number of treatments. Most of the Lattice designs will be either B.I.B. or P.B.I.B designs. However, there are exceptions. The construction of Lattice designs is based on the correspondence between the treatments and the treatment combinations of a factorial experiment. The number of treatments $(\mathrm{t})$ and block size $(\mathrm{k})$ are related. The form of relationship can be

$$
\mathrm{t}=\mathrm{k}^{2} \text { or } \mathrm{t}=\mathrm{k}^{3} \text { or } \mathrm{t}=\mathrm{k}(\mathrm{k}+1)
$$

The Lattice designs though restricted by the above relationships between $\mathrm{t}$ and $\mathrm{k}$ are an important class of designs when the treatment number is very large. In breeding experiments it is not surprising to find the number 
of treatments being more than 60 . In varietal trials where a large number of varieties are screened, Lattice designs are most appropriate.

A Lattice Square $\left(t=k^{2}\right)$ constructed by arranging the treatments in a square $(\mathrm{kx} \mathrm{k})$ where the block size is $\mathrm{k}$. Thus, there will be $\mathrm{k}$ blocks of size $\mathrm{k}$. This is one full replicate of the Lattice Square Design. For a balanced Lattice there will be $(k+1)$ such replicates. As for I.B.D., catalogues are available for Lattice designs. If fewer than $(\mathrm{k}+1)$ replicates are used, the designs will be called partially balanced Lattices comparable to P.B.I.B. designs.

Lattice designs can be classified on the basis of number of treatments $(\mathrm{t})$, block size $(\mathrm{k})$ and the number of replicate squares used. In addition to square Lattices, cubic Lattices, Lattice Square and rectangular Lattices are available.

If the number of treatments is given by $\mathrm{t}=\mathrm{k}^{3}$, we will need $\mathrm{k}^{2}$ blocks of size $\mathrm{k}$. Such designs are called three dimensional Lattice designs. Higher dimensional Lattice designs such as $\mathrm{t}=\mathrm{k}^{5}$ can be constructed using the correspondence to the treatment combinations of a $\mathrm{k}^{5}$ factorial.

\section{Confounding and Fractional Factorials}

In large factorial experiments, even with 2 levels of a factor if there are a large number of factors investigated, the number of treatment combinations becomes extremely large. It will be impossible to find homogeneous blocks large enough to accommodate all treatment combinations. For instance with 6 factors at 2 levels each, there will be 64 treatment combinations. It the block sizes are small, we need to sacrifice information on the higher order interactions, to be able to estimate main effects and lower order interactions. The estimates of the interactions that are Sacrificed cannot be distinguished from the block effect. This phenomenon is called confounding.

Different effects can be confounded in different replicates leading to partially confounded designs. When the number of factors investigated increases, the number of treatment combinations increase rapidly. For instance in industrial experiments where optimum levels of different chemicals are investigated, it is possible that more than 5- 8 factors are involved. For an experiment with 8 factors at only 2 levels each there will be 256 treatment combinations. It may therefore be impossible to have even a single replicate of all the treatment combinations in an experiment. This leads to experiments using only a fraction of the treatment combinations (Eg. $1 / 2$ fraction, $1 / 4$ fraction, $1 / 8$ fraction etc for a $2^{\mathrm{k}}$ factorial) in the entire experiment. Such experiments are called fractional factorials. Information on main effects and lower order interactions are obtained by sacrificing information on higher order interactions as in confounded factorial experiments.

\section{References}

1. Hinkelman K, Kempthorne O (2005) Design and analysis of experiments, Advanced Experimental Design. In: Walter A Shewhart and Samuel S Wilks (Eds.), Volume 2, John Wiley and Sons, New York, USA.

2. Thattil RO, Nsowah-Nuomah NNN (2013) Advanced Topics in Design and Analysis of Experiments (2nd edn), Postgraduate Institute of Agriculture, University of Peradeniya, Sri Lanka. 